\title{
Coloured and Gray Scale Image Steganography using Block Level DWT DCT Transformation
}

\author{
Anuradha Goswami \\ M.tech Student \\ GITS, Udaipur, India
}

\author{
Sarika Khandelwal \\ Assistant Professor \\ GITS, Udaipur, India
}

\begin{abstract}
Piracy is a big threat to the original contents. This multi-model approach interleaves some frequency domain watermarking strategies to counteract shortcomings of one strategy by the advantages of other. The original image is divided into spatially disjoint blocks. Watermarking is a way of embedding a mark that hides information in a multimedia carrier. Major issues in Image watermarking are to increase the robustness visual attacks. Image The Proposed algorithm presents Hybrid Discrete wavelet transform and Discrete cosine transform based watermarking technique to obtain increased imperceptibility and robustness compared to DWT based watermarking technique. The idea of inserting embedding image in combined transform is based on fact that joint transform eliminates drawback of each other and secret information is hidden in High frequency area which signify the noise in image and characteristics of image is not changed.
\end{abstract}

\section{Keywords}

Steganography, Watermarking, Invisible hiding

\section{INTRODUCTION}

Currently a lot of technique support is incorporated with the watermarking systems to increase the strength of steganography system. Devices are used for reading and writing of digital copies to embed or check the watermarks while creating the copies [1]. They can check that if watermark is found then this reader can reject copying of this disc. It is also a good idea to embed the watermark as soon as any digital information is created as done by new age digital cameras [2]. They embed the camera id, owner id, date and time into the image. But all these applications requires support of firm anti piracy laws to sustain these technologies since no one is going to incorporate these extra systems in his device specially if he can be caught making illegal use of it. Secret data can be hidden into an unimportant medium so no illegitimate person will expect its existence into this medium this is called steganography. Another way is to deform the secret data into an unusable or non-interpretable form is called cryptography [3]. The process of reverting cipher text back to its original form is called decryption [4]. Steganography system urges to secure embedding of a large amount of information, with no visible degradation to the cover object but watermarking system, however, embeds information that stick to the cover object so hard that it could not be removed or altered without making the cover object entirely unusable [5]. In a digital image, information can be inserted directly into every bit of image information or the more busy areas of an image can be calculated so as to hide such messages in less perceptible parts of an image [6]. Least significant bit (LSB) insertion is a common, simple approach to embedding information in a cover image. if a bit 1 is hidden in pixel value then pixel value is converted into binary. For Example if pixel value is 10 then (10) $10 \rightarrow(00001010) 2$. The secret bit is embedded by replacing of lsb bit of pixel value. So for example 00001010 is converted into 00001011 which is 11 in decimal. So in LSB-1 technique maximum value difference is 1 and effect is not perceptible by human eyes. With a wellchosen image, one can even hide the message in the least as well as second to least significant bit and still not see the difference. In the above example, consecutive bytes of the image data - from the first byte to the end of the message are used to embed the information [7].

\section{PROPOSED WORK}

It is concluded from the research that many embedding image are embed in the HL sub band (Vertical Sub band) and HH sub band (Diagonal sub-band) of the detail coefficients of wavelet transform for maximize robustness against statistical attacks and robustness against visual attacks or imperceptibility[8]. The detailed embedding procedure of proposed technique is explained as following.

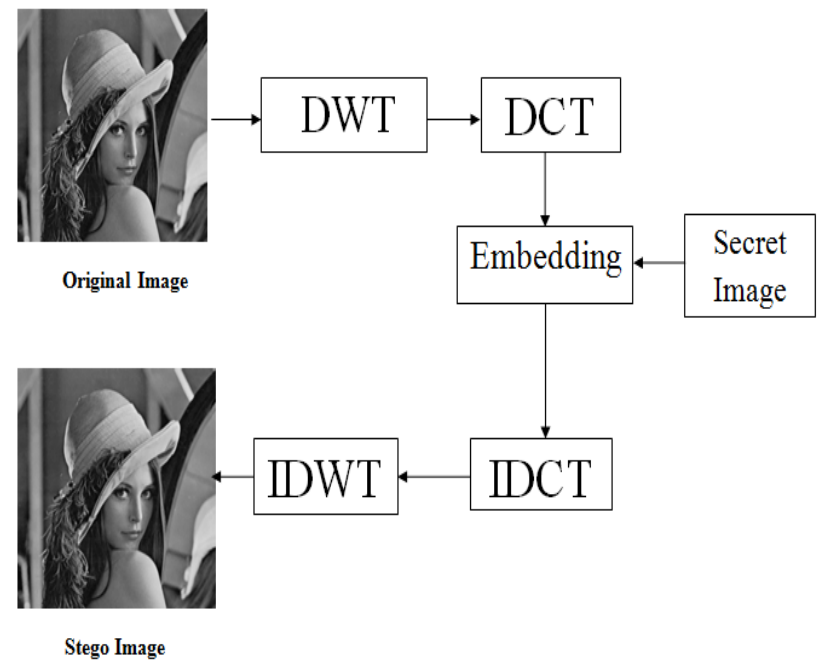

Fig 1: Embedding Process

Step 1. Discrete Wavelet Transformation[9] is applied on cover image for decomposing into sub-bands.

Step 2. Discrete Wavelet Transformation is applied again on all above sub-bands for decomposing into 16 sub-bands and four $\mathrm{HH} 2$ (HH sub-bands at level 2) sub-bands are selected.

Step 3. Discrete Wavelet Transformation is applied again on selected four HH2 sub-bands for decomposing into 16 subbands.

Step 4. four HH sub-bands are selected.

Step 4.Perform Discrete Cosine Transform[10] at all selected sub bands. 
Step 5. Embedding image is converted into binary format and bits of DCT coefficients of above selected sub band are modified with bits of secret image.

Step 6.Applyinverse Discrete Cosine Transform[11] on each sub band.

Step 7. Apply Discrete Integer Wavelet Transform[12] to get secret image embedded image.

\section{EXPERIMENTS \& RESULTS}

The Experiments of Proposed Hybrid IWT-DCT image steganography technique is performed on host images of Lena, Cameraman, Barbara, Pepper \& Baboon of size of 512x512 pixels each on gray scale and coloured Images . A secret image image of size of $32 \times 32$ binary images as shown in the figure is embedded as secret image in above host images.

Table 1: Results of digital image steganography in Gray Scale image 512x512 using proposed hybrid DCT-DWT technique

\begin{tabular}{|c|c|c|c|}
\hline Sno. & Image Name & Watermark & $\begin{array}{l}\text { Proposed } \\
\text { DWT+DCT } \\
\text { PSNR }\end{array}$ \\
\hline 1 & Leena & AB & 44 \\
\hline 2 & Cameraman & AB & 51 \\
\hline 3 & Barbara & AB & 49 \\
\hline 4 & Baboon & AB & 47 \\
\hline
\end{tabular}

Table 2: Results of digital image steganography algorithm compared to existing Mingwei Zhao \& Yanzhong Dang[13]

\begin{tabular}{|c|c|c|c|c|}
\hline Sno. & $\begin{array}{l}\text { Image } \\
\text { Name } \\
\text { (Coloure } \\
\text { d image) }\end{array}$ & $\begin{array}{l}\text { Water } \\
\text { mark }\end{array}$ & $\begin{array}{l}\text { PSNR Mingwei } \\
\text { \& Yanzhong }\end{array}$ & $\begin{array}{l}\text { Proposed } \\
\text { (DWT+DCT) } \\
\text { PSNR }\end{array}$ \\
\hline 1 & Leena & AB & 48 & 50 \\
\hline 2 & Baboon & AB & 46 \\
& \multicolumn{2}{|c|}{4} & \\
\hline
\end{tabular}

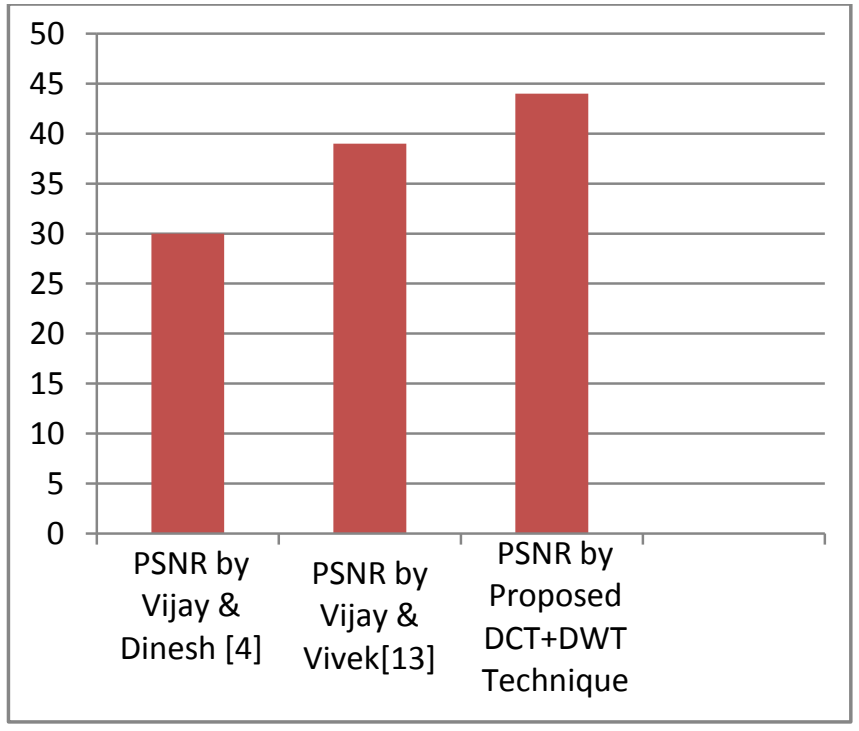

Fig 2: Chart For Gray scale Image PSNR Existing v/s. Proposed

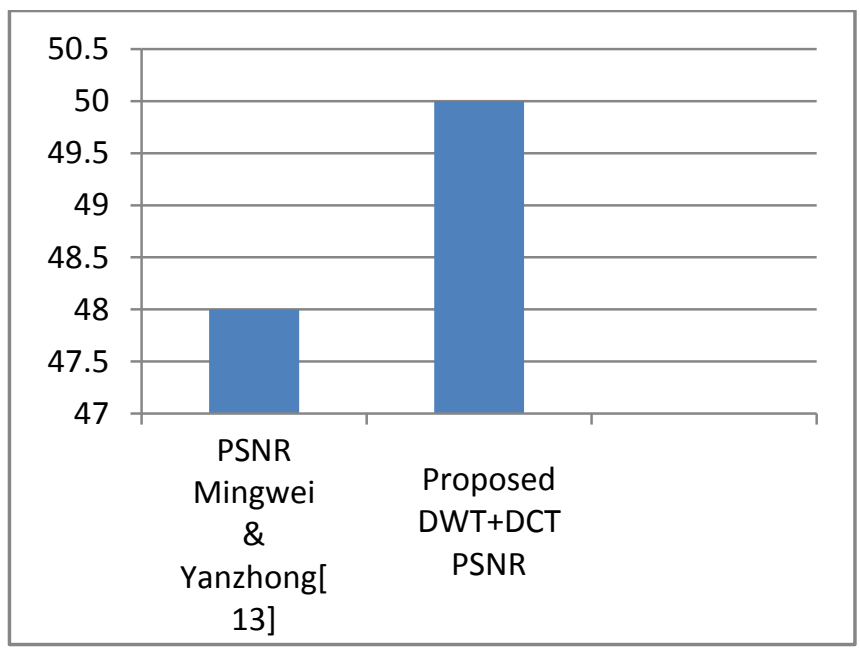

Fig 3: Chart For Coloured Image PSNR Existing v/s. Proposed

\section{CONCLUSION}

From the results it is derived that proposed technique achieved PSNR value 44 while vijay \& dinesh method provide 29 \& vijaya \& vivek 's method provide PSNR 39.2. And for Coloured images proposed technique achieved PSNR value 50 while Mingwei \& Yanzhong method provide 48.So proposed method achieved PSNR more than existing methods Mingwei \& Yanzhong [13], vijay \& dinesh [4] \& vijaya \& vivek [14]. PSNR signify robustness against visual attack for invisible steganography. So proposed technique will have higher imperceptibility or higher robustness against visual attacks. In the proposed technique the watermark is embedded in $\mathrm{HH}$ sub band or edge and noise information not in characteristics and shapes information of cover image.

\section{REFERENCES}

[1] Anshul Khairwal, Kumar Abhishek, Surya Prakash, Tej Pratap, "A Comprehensive Study of Various Biometric Identification Techniques", in International Conference on ICCCNT, IEEE, 2012.

[2] Abbas Cheddad A., Joan Condell, Kevin Curran, Paul Mc Kevitt, "Digital image steganography: Survey and 
analysis of current methods", International Journal of Signal Processing, Elsevier pp:727-752, 2010.

[3] C.C. Chang, Y.Z. Wang and C.S. Chan, "An Efficient Probability-Based $t$ out of $n$ Secret Image Sharing Scheme", Second International Conference on Future Generation Communication and Networking Symposia, 2008. FGCNS '08., vol.3, pp.121-124, 2008.

[4] Vijay Kumar, Dinesh Kumar, "Performance Evaluation of DWT Based Image Steganography", International Conference on Advance Computing, IEEE, 2010.

[5] Gheorghita Ghinea, Adel Almohammad, "Image Steganography and Chrominance Components", International Conference on Computer and Information Technology, IEEE, pp: 996- 1001, 2010.

[6] O.El Safy, H.H. Zayed and A.El Dessouki, "A Adoptive Steganographic Technique based On Integer Wavelet Transform", International Conference on Networking and Media Convergence, IEEE, pp:111-117, 2009.

[7] Elham Ghasemi, Bahram ZahirAzami, Jamshid Shanbehzadeh, "A Steganographic method based on Integer Wavelet Transform and Genetic Algorithm", International Conference on Communications and Signal Processing, IEEE, pp:42-44, 2010.

[8] Michiharu Niimi, Hideki Noda, Bruce Segee, " Robust BPCS Steganography against the Visual Attack ", International Conference on Communications and Signal Processing, IEEE, 2007.
[9] Tao Zhang, Zhaohui Li, Peipei Shi, "Statistical Analysis Against improved BPCS Steganography", International Conference on Advanced Computer Control, IEEE, pp. 237- 240, 2010

[10] Peipei Shi Zhaohui Li Tao Zhang, "A technique of improved steganography text based on chaos and BPCS", International Conference on Advanced Computer Control, IEEE, pp. 232- 236, 2010.

[11] Julio Lopez, Raul Martinez, Mariko Nakhano and Kazuhiko , "Detection of BPCS Steganography Using SMWCF Steganalysis and SVM", International Symposium on Information Theory and its Applications, IEEE, 2008.

[12] Xie Yong, Zhu Zhou, "Analysis and Comparison of Holographic and Traditional Digital Image Watermarking in DWT Domain", The 7th International Conference on Computer Science \& Education (ICCSE 2012), IEEE, July 14-17, 2012.

[13] M. Zhao and Y. Dang, "Color Image Copyright Protection Digital Watermarking Algorithm Based on DWT DCT", IEEE,2008.

[14] Vijaya K. Ahire, Vivek Kshirsagar ,"Robust Watermarking Scheme Based on Discrete Wavelet Transform (DWT) and Discrete Cosine Transform (DCT) for Copyright Protection of Digital Images", IJCSNS International Journal of Computer Science and Network Security, VOL.11 No.8, August 2011. 